University of Texas at El Paso

ScholarWorks@UTEP

$10-2013$

\title{
Picture Fuzzy Sets - a new concept for computational intelligence problems
}

\author{
Bui Cong Cuong \\ Vietnam Academy of Science and Technology, bccuong@gmail.com \\ Vladik Kreinovich \\ The University of Texas at El Paso, vladik@utep.edu
}

Follow this and additional works at: https://scholarworks.utep.edu/cs_techrep

Part of the Computer Sciences Commons

Comments:

Technical Report: UTEP-CS-13-66

Published in Proceedings of the Third World Congress on Information and Communication Technologies WICT'2013, Hanoi, Vietnam, December 15-18, 2013, pp. 1-6.

\section{Recommended Citation}

Cuong, Bui Cong and Kreinovich, Vladik, "Picture Fuzzy Sets - a new concept for computational intelligence problems" (2013). Departmental Technical Reports (CS). 809.

https://scholarworks.utep.edu/cs_techrep/809

This Article is brought to you for free and open access by the Computer Science at ScholarWorks@UTEP. It has been accepted for inclusion in Departmental Technical Reports (CS) by an authorized administrator of ScholarWorks@UTEP. For more information, please contact Iweber@utep.edu. 


\section{Picture Fuzzy Sets - a new concept for computational intelligence problems}

\author{
Bui Cong Cuong* \\ Institute of Mathematics \\ Vietnam Academy of Science and Technology \\ 18 Hoang Quoc Viet, Cau Giay, Hanoi, Vietnam \\ bccuong@gmail.com
}

\author{
Vladik Kreinovich \\ Department of Computer Science \\ University of Texas at El Paso \\ El Paso, TX 79968, USA \\ vladik@cs.utep.edu
}

\begin{abstract}
Since Zadeh introduced fuzzy sets in 1965, a lot of new theories treating imprecision and uncertainty have been introduced. Some of these theories are extensions of fuzzy set theory, other try to handle imprecision and uncertainty in different way. In this paper, we introduce a new notion of picture fuzzy sets (PFS), which are directly extensions of fuzzy sets and of intuitonistic fuzzy sets (Atanassov). Then some operations on picture fuzzy sets are defined and some properties of these operations are considered. Here the basic preliminaries of PFS theory are presented.
\end{abstract} set.

Keywords: Picture fuzzy set, Operation, Intuitionistic fuzzy

\section{INTRODUCTION}

Computational Intelligence is a set of Nature-inspired computational methodologies and approaches based on the achievements of mathematics, computer science, artificial intelligence, to address complex problems of the real world applications to which traditional methodologies and approaches are ineffective or infeasible.

Naturally, researches about operators and computing processes in fuzzy sets and fuzzy logic promised to contribute to Computational Intelligence, especially to the real word problems, in which there are many vague or uncertainty factors.

Since Zadeh introduced fuzzy sets (FS) in 1965, a lot of new theories treating imprecision and uncertainty have beeen introduced. Some of these theories are extensions of fuzzy set theory, other try to handle imprecision and uncertainty in different way. Intuitionistic fuzzy sets (IFS) were introduced by Atanassov[1] in 1983 constitute a generalization of the notion of a fuzzy set .When fuzzy set give the degree of membership of an element in a given set, intuitionistic fuzzy sets give a degree of membership and a degree of non-membership.

Definition 1:[1] A intuitionistic fuzzy set $A$ on a universe $X$ is an object of the form

$$
A=\left\{\left(x, \mu_{A}(x), v_{A}(x)\right) \mid x \in X\right\},
$$

where $\mu_{A}(x)(\in[0,1])$ is called the "degree of membership of $\mathrm{x}$ in $\mathrm{A}$ ", $v_{A}(u)(\in[0,1])$ is called the "degree of non- membership of $\mathrm{x}$ in A", and where $\mu_{\mathrm{A}}$ and $v_{\mathrm{A}}$ satisfy the following condition:

$$
(\forall x \in X) \quad\left(\mu_{A}(x)+v_{A}(x) \leq 1\right) .
$$

In this paper, let $\operatorname{IFS}(X)$ denote the set of all the intuitionistic fuzzy set IFSs on a universe $X$.

A generalization of fuzzy sets and intuitionistic fuzzy sets are the following notion of picture fuzzy sets .

Definition 2: A picture fuzzy set $A$ on a universe $X$ is an object of the form

$$
A=\left\{\left(x, \mu_{A}(x), \eta_{A}(x), v_{A}(x)\right) \mid x \in X\right\},
$$

where $\mu_{A}(x)(\in[0,1])$ is called the "degree of positive membership of $\mathrm{x}$ in $\mathrm{A}$ ”, $\quad \eta_{\mathrm{A}}(x)(\in[0,1])$ is called the "degree of neutral membership of $\mathrm{x}$ in $\mathrm{A}$ " and $v_{A}(u)(\in[0,1])$ is called the "degree of negative membership of $\mathrm{x}$ in A", and where $\mu_{\mathrm{A}}, \eta_{\mathrm{A}}$ and $v_{\mathrm{A}}$ satisfy the following condition:

$$
(\forall x \in X) \quad\left(\mu_{A}(x)+\eta_{A}(x)+v_{A}(x) \leq 1\right) \quad .
$$

Then for $x \in X, 1-\left(\mu_{A}(x)+\eta_{A}(x)+v_{A}(x)\right)$ could be called the "degree of refusal membership of $x$ in A".

Basically, picture fuzzy sets based models may be adequate in situations when we face human opinions involving more answers of type: yes, abstain, no , refusal.

Voting can be a good example of such a situation as the human voters may be divided into four group of those who: vote for, abstain, vote against, refusal of the voting.

Let $P F S(X)$ denote the set of all the picture fuzzy set PFSs on a universe $X$.

Definition 3: For every two PFSs A and B, the union, intersection and complement are defined as follows:

$$
\begin{array}{ll}
\text { - } & A \subseteq B \text { iff }\left(\forall x \in X, \mu_{A}(x) \leq \mu_{B}(x)\right. \text { and } \\
& \left.\eta_{A}(x) \leq \eta_{B}(x) \text { and } \quad v_{A}(x) \geq v_{B}(x)\right) \\
\text { - } & A=B \quad \text { iff }(A \subseteq B \text { and } B \subseteq A)
\end{array}
$$


$A \cup B=\left\{\left(x, \max \left(\mu_{A}(x), \mu_{B}(x)\right), \min \left(\eta_{A}(x), \eta_{B}(x)\right)\right.\right.$,

- $\left.\left.\min \left(v_{A}(x), v_{B}(x)\right)\right) \mid x \in X\right\}$

- $A \cap B=\left\{\left(x, \min \left(\mu_{A}(x), \mu_{B}(x)\right), \min \left(\eta_{A}(x), \eta_{B}(x)\right)\right.\right.$,

$\left.\left.\max \left(v_{A}(x), v_{B}(x)\right)\right) \mid x \in X\right\}$

- $\operatorname{co} A=\bar{A}=\left\{\left(x, v_{A}(x), \eta_{A}(x), \mu_{A}(x)\right) \mid x \in X\right\}$.

Now we will introduce a generalisation of intervalvalued fuzzy set $A$. Here the int $([0,1])$ stands for the set of all closed subinterval of $[0,1]$.

Definition 4: Let $\left[a_{1}, b_{1}\right],\left[a_{2}, b_{2}\right] \in \operatorname{int}([0,1])$. We define

$$
\begin{aligned}
& {\left[a_{1}, b_{1}\right] \leq\left[a_{2}, b_{2}\right], \quad \text { iff } a_{1} \leq a_{2}, b_{1} \leq b_{2}} \\
& {\left[a_{1}, b_{1}\right] \precsim\left[a_{2}, b_{2}\right] \quad \text { iff } a_{1} \leq a_{2}, b_{1} \geq b_{2}} \\
& {\left[a_{1}, b_{1}\right]=\left[a_{2}, b_{2}\right], \quad \text { iff } a_{1}=a_{2}, b_{1}=b_{2}} \\
& {\left[a_{1}, b_{1}\right] \vee\left[a_{2}, b_{2}\right]=\left[a_{1} \vee a_{2}, b_{1} \vee b_{2}\right]} \\
& {\left[a_{1}, b_{1}\right] \wedge\left[a_{2}, b_{2}\right]=\left[a_{1} \wedge a_{2}, b_{1} \wedge b_{2}\right]} \\
& {\left[a_{1}, b_{1}\right] \times\left[a_{2}, b_{2}\right]=\left[a_{1} \times a_{2}, b_{1} \times b_{2}\right]}
\end{aligned}
$$

Let $\alpha \geq 0$. We define $\alpha\left[a_{1}, b_{1}\right]=\left[\alpha a_{1}, \alpha b_{1}\right]$.

If $0 \leq \alpha \leq 1$, then $\alpha\left[a_{1}, b_{1}\right] \in \operatorname{int}([0,1])$.

Definition 5: An interval-valued picture fuzzy set $A$ on a universe $X$ (IvPFS, in short) is an object of the form

$$
A=\left\{\left(x, M_{A}(x), L_{A}(x), N_{A}(x)\right) \mid x \in X\right\},
$$

where $\quad M_{A}(x)=\left[M_{A L}(x), M_{A U}(x)\right] \in \operatorname{int}([0,1])$,

$$
\begin{aligned}
& L_{A}(x)=\left[L_{A L}(x), L_{A U}(x)\right] \in \operatorname{int}([0,1]), \\
& N_{A}(x)=\left[N_{A L}(x), N_{A U}(x)\right] \in \operatorname{int}([0,1]),
\end{aligned}
$$

satisfy the following condition:

$$
(\forall x \in X) \quad\left(\sup M_{A}(x)+\sup L_{A}(x)+\sup N_{A}(x) \leq 1\right) \quad .
$$

Let $\operatorname{IvPFS}(X)$ denote the set of all the interval-valued picture fuzzy set IvPFSs on a universe $X$.

Definition 6: For every two IvPFSs A and B, the inclusion, union, intersection and complement are defined as follows:

\footnotetext{
$A \subseteq B$ iff $(\forall x \in X)\left(\left(M_{A}(x) \leq M_{B}(x)\right)\right.$ and

$\left(L_{A}(x) \leq L_{B}(x)\right)$ and $\left.\left(N_{A}(x) \geq N_{B}(x)\right)\right)$

- $A=B$ iff $A \subseteq B$ and $B \subseteq A$
}

$$
\begin{aligned}
& A \cup B=\left\{\left(x, M_{A}(x) \vee M_{B}(x), L_{A}(x) \wedge L_{B}(x),\right.\right. \\
& \left.\left.N_{A}(x) \wedge N_{B}(x)\right) \mid x \in X\right\} \\
& A \cap B=\left\{\left(x, M_{A}(x) \wedge M_{B}(x), L_{A}(x) \wedge L_{B}(x),\right.\right. \\
& \left.\left.N_{A}(x) \vee N_{B}(x)\right) \mid x \in X\right\}
\end{aligned}
$$

where $\vee$ and $\wedge$ stand for max and min operators respectively

- $\quad \operatorname{co} A=\bar{A}=\left\{\left(x, N_{A}(x), L_{A}(x), M_{A}(x)\right) \mid x \in X\right\}$.

Definition 7: Let $X_{1}$ and $X_{2}$ be two universums and let $A=\left\{\left(x, \mu_{A}(x), \eta_{A}(x), v_{A}(x)\right) \mid x \in X_{1}\right\}$ and $B=\left\{\left(y, \mu_{B}(y), \eta_{B}(y), v_{B}(y)\right) \mid y \in X_{2}\right\}$ be two PFSs .

We define the Cartesian product of these two PFS's

$$
\begin{aligned}
\text { - } \quad A \times_{1} B & =\left\{\begin{array}{l}
\left((x, y), \mu_{A}(x) \cdot \mu_{B}(y), \eta_{A}(x) \cdot \eta_{B}(y),\right. \\
\left.v_{A}(x) \cdot v_{B}(y)\right) \mid x \in X_{1}, y \in X_{2}
\end{array}\right\} \\
\text { - } \quad A \times_{2} B & =\left\{\begin{array}{l}
\left((x, y), \mu_{A}(x) \wedge \mu_{B}(y), \eta_{A}(x) \wedge \eta_{B}(y),\right. \\
\left.v_{A}(x) \vee v_{B}(y)\right) \mid x \in X_{1}, y \in X_{2}
\end{array}\right\}
\end{aligned}
$$

These definitions are valid.

Proof: See [7] .

Definition 8: Let A be an IvPFS over $X_{1}$ and $\mathrm{B}$ be an IvPFS over $X_{2}$.We define:

$$
\begin{aligned}
& A \times_{1} B=\left\{\left((x, y),\left(M_{A}(x) \times M_{B}(y), L_{A}(x) \times L_{B}(y),\right.\right.\right. \\
& \left.\left.N_{A}(x) \times N_{B}(y)\right) \mid x \in X_{1}, y \in X_{2}\right\} \\
& A \times_{2} B=\left\{\left((x, y),\left(M_{A}(x) \wedge M_{B}(y), L_{A}(x) \wedge L_{B}(y),\right.\right.\right. \\
& \left.\left.N_{A}(x) \vee N_{B}(y)\right) \mid x \in X_{1}, y \in X_{2}\right\}
\end{aligned}
$$

The proof of the validity of these operators is given in [7]. Some of the defined operations are extensions of the FS operations [6], [11], [12] and of some of operations for IFSs and IvIFSs [1 -4], [10] and [13].

\section{SOME OPERATIONS ON PFS}

Now we consider some propeties of the defined operations on PFS.

Proposition 1: For every PFS's A,B,C

(a) If $A \subseteq B$ and $B \subseteq C$ then $A \subseteq C$;

(b) $\overline{\overline{(A)}}=A$;

(c) Operations $\cap$ and $\cup$ are commutative, associative, and distributive; 
(d) Operations $\cap$, Co and $\cup$ satisfy the law of De Morgan.

Proof: See [7].

Proposition 2: For every three universums $X_{1}, X_{2}, X_{3}$ and four PFS's ( or four IvPFS's) $A, B$ (over $X_{1}$ ), $C$ (over $X_{2}$ ), $D$ ( over $X_{3}$ ):
(a) $A \times_{1} C=C \times_{1} A$;
(b) $\quad\left(\left(A \times_{1} C\right) \times_{1} D=A \times_{1}\left(C \times_{1} D\right)\right.$;
(c) $(A \cup B) \times \times_{1} C=\left(A \times_{1} C\right) \cup\left(B \times \times_{1} C\right)$;
(d) $(A \cap B) \times \times_{1} C=\left(A \times_{1} C\right) \cap\left(B \times{ }_{1} C\right)$.

Proof: See [7].

Proposition 3: For the Cartesian produce of PFSs

$A \times_{2} B=\left\{\begin{array}{l}\left((x, y), \mu_{A}(x) \wedge \mu_{B}(y), \eta_{A}(x) \wedge \eta_{B}(y),\right. \\ \left.v_{A}(x) \vee v_{B}(y)\right) \mid x \in X_{1}, y \in X_{2}\end{array}\right\}$

analogously, we have

(a) $A \times{ }_{2} C=C \times{ }_{2} A$;

(b) $\quad\left(\left(A \times_{2} C\right) \times{ }_{2} D=A \times_{2}\left(C \times_{2} D\right)\right.$;

(c) $(A \cup B) \times{ }_{2} C=\left(A \times{ }_{2} C\right) \cup\left(B \times{ }_{2} C\right)$;

(d) $(A \cap B) \times{ }_{2} C=\left(A \times{ }_{2} C\right) \cap\left(B \times{ }_{2} C\right)$

Proof: See [8].

Proposition 4: For every three universums $X_{1}, X_{2}, X_{3}$ and four IvPFS's $A, B$ (over $X_{1}$ ), $C$ (over $X_{2}$ ), $D$ ( over $\left.X_{3}\right)$. For the Cartesian produce of IvPFSs

$A \times_{2} B=\left\{\begin{array}{l}\left((x, y), M_{A}(x) \wedge M_{B}(y), L_{A}(x) \wedge L_{B}(y),\right. \\ \left.N_{A}(x) \vee N_{B}(y)\right) \mid x \in X_{1}, y \in X_{2}\end{array}\right\}$

analogously, we have:

(a) $A \times{ }_{2} C=C \times{ }_{2} A$;

(b) $\quad\left(\left(A \times_{2} C\right) \times{ }_{2} D=A \times_{2}\left(C \times_{2} D\right)\right.$;

(c) $(A \cup B) \times{ }_{2} C=\left(A \times{ }_{2} C\right) \cup\left(B \times{ }_{2} C\right)$;

(d) $(A \cap B) \times{ }_{2} C=\left(A \times{ }_{2} C\right) \cap\left(B \times{ }_{2} C\right)$

Proof: See [8].

\section{A. Distance between picture fuzzy sets}

Distance between fuzzy sets and distance between intuitionistic fuzzy sets were defined in fuzzy literature and have been applied in various problems [10],[11]. In this subsection some extensions of the distances between intuitionistic fuzzy sets of Szmith and Kacprzyk [13] are presented.

Definition 9: Distances for two picture fuzzy sets $A$ and $B$ in $X=\left\{x_{1}, x_{2}, \ldots, x_{n}\right\}$ are:

- $\quad$ The normalized Hamming distance $d_{P}(A, B)$

$$
d_{P}(A, B)=\frac{1}{n} \sum_{i=1}^{n}\left(\left|\mu_{A}\left(x_{i}\right)-\mu_{B}\left(x_{i}\right)\right|+\left|\eta_{A}\left(x_{i}\right)-\eta_{B}\left(x_{i}\right)\right|+\left|v_{A}\left(x_{i}\right)-v_{B}\left(x_{i}\right)\right|\right)
$$

- $\quad$ The normalized Euclidean distance $e_{P}(A, B)$

$e_{P}(A, B)=\sqrt{\left.\frac{1}{n} \sum_{i=1}^{n}\left(\left(\mu_{A}\left(x_{i}\right)\right)-\mu_{B}\left(x_{i}\right)\right)^{2}+\left(\eta_{A}\left(x_{i}\right)-\eta_{B}\left(x_{i}\right)\right)^{2}+\left(v_{A}\left(x_{i}\right)-v_{B}\left(x_{i}\right)\right)^{2}\right)}$

Example 1: Let us consider picture fuzzy sets $A, B, C$ in $X=\left\{x_{1}, x_{2}, x_{3}\right\}, A$ full description of picture fuzzy set

$\begin{array}{ll}A \text {, i.e. } & A=\left\{\left(\mu_{A}\left(x_{1}\right), \eta_{A}\left(x_{1}\right), v_{A}\left(x_{1}\right)\right) / x_{1},\left(\mu_{A}\left(x_{2}\right), \eta_{A}\left(x_{2}\right), v_{A}\left(x_{2}\right)\right) / x_{2},\right. \\ & \left.\left(\mu_{A}\left(x_{3}\right), \eta_{A}\left(x_{3}\right), v_{A}\left(x_{3}\right)\right) / x_{3}\right\}\end{array}$

For example,

$$
\begin{aligned}
& A=\left\{(0.8,0.1,0) / x_{1},(0.4,0.2,0.3) / x_{2},(0.5,0.3,0) / x_{3}\right\} \\
& B=\left\{(0.3,0.3,0.2) / x_{1},(0.7,0.1,0.1) / x_{2},(0.4,0.3,0.2) / x_{3}\right\} \\
& C=\left\{(0.3,0.4,0.1) / x_{1},(0.6,0.2,0.1) / x_{2},(0.4,0.3,0.1) / x_{3}\right\} .
\end{aligned}
$$

Then $d_{P}(A, B)=0.6, d_{P}(B, C)=0.5 / 3, d_{P}(A, C)=0.5$

$e_{P}(A, B)=\sqrt{0.52 / 3}, e_{P}(B, C)=\sqrt{0.05 / 3} \quad, e_{P}(A, C)=\sqrt{0.15} \quad$.

\section{B. Convex combination of PFS}

Convex combination is an important operation in mathematics, which is an useful tool on convex analysis, linear spaces and convex optimization. In this sub-section convex combination firstly is defined with some simple propositions. This new operation should be more considered in the other papers.

Definition 10: Let $A, B$ be a PFS on $X$. Let $\theta$ be a real number such that $0 \leq \theta \leq 1$.

For each $\theta$, the convex combination of $A$ and $B$ is defined as follows:

$C_{\theta}(A, B)=\left\{\left(x, \mu_{C_{\theta}}(x), \eta_{C_{\theta}}(x), v_{C_{\theta}}(x)\right) \mid x \in X\right\}$ where

$\forall x \in X, \mu_{C_{\theta}}(x)=\theta \cdot \mu_{A}(x)+(1-\theta) \cdot \mu_{B}(x)$,

$\forall x \in X, \eta_{C_{\theta}}(x)=\theta \cdot \eta_{A}(x)+(1-\theta) \cdot \eta_{B}(x)$,

$\forall x \in X, v_{C_{\theta}}(x)=\theta \cdot v_{A}(x)+(1-\theta) \cdot v_{B}(x)$.

Analogously, the convex combination of IvPFSs is defined. 
Definition 11: Let $A, B$ be two IvPFS on $X$. Let $\theta$ be a real number such that $0 \leq \theta \leq 1$.

For each $\theta$, the convex combination of $A$ and $B$ is defined as follows:

$C_{\theta}(A, B)=\left\{\left(x, M_{C_{\theta}}(x), L_{C_{\theta}}(x), N_{C_{\theta}}(x)\right) \mid x \in X\right\}$

where

$\forall x \in X, M_{C_{\theta}}(x)=\left[\left(M_{C_{\theta} L}(x)=\theta \cdot M_{A L}(x)+(1-\theta) \cdot M_{B L}(x)\right)\right.$, $\left.\left(M_{C_{\theta} U}(x)=\theta \cdot M_{A U}(x)+(1-\theta) \cdot M_{B U}(x)\right)\right]$,

$\forall x \in X, L_{C_{\theta}}(x)=\left[\left(L_{C_{\theta} L}(x)=\theta \cdot L_{A L}(x)+(1-\theta) . L_{B L}(x)\right)\right.$,

$\left.\left(L_{C_{\theta} U}(x)=\theta \cdot L_{A U}(x)+(1-\theta) \cdot L_{B U}(x)\right)\right]$,

$\forall x \in X, N_{C_{\theta}}(x)=\left[\left(N_{C_{\theta} L}(x)=\theta \cdot N_{A L}(x)+(1-\theta) \cdot N_{B L}(x)\right)\right.$,

$\left.\left(N_{C_{\theta} U}(x)=\theta \cdot N_{A U}(x)+(1-\theta) \cdot N_{B U}(x)\right)\right]$.

Proposition 5: Let $A, B$ be two PFS (or two IvPFS )

on $X$. Let $\theta$ be a real number such that $0 \leq \theta \leq 1$.Then

- If $\theta=1$, then $C_{\theta}(A, B)=A$ and if $\theta=0$, then $C_{\theta}(A, B)=B$;

- If $A \subseteq B$, then $\forall \theta, \quad A \subseteq C_{\theta}(A, B) \subseteq B \quad$;

- If $(A \supseteq B) \&\left(\theta_{1} \geq \theta_{2}\right)$, then $\quad C_{\theta_{1}}(A, B) \supseteq C_{\theta_{2}}(A, B)$.

Proof: See [8].

Proposition 6: Let $A, B, D$ be three PFS (or three IvPFS) on $X$. Let $\theta$ be a real number such that $0 \leq \theta \leq 1$. Then

- $C_{\theta}(A \cap B, D)=C_{\theta}(A, D) \cap C_{\theta}(B, D)$;

- $C_{\theta}(A \cup B, D)=C_{\theta}(A, D) \cup C_{\theta}(B, D)$.

Proof: See [8].

We can define some simple aggregation operators on PFS.

Definition 12: Let $\left\{A_{1}, \ldots, A_{n}\right\}$ be a vector of PFSs on $X$, where $A_{i}=\left\{\left(x, \mu_{A_{i}}(x), \eta_{A_{i}}(x), v_{A_{i}}(x)\right) \mid x \in X\right\}, \mathrm{i}=1,,,,, \mathrm{n}$

Let $\alpha=\left\{\alpha_{1}, \ldots, \alpha_{n}\right\}$ be a weighted vector such that $0 \leq \alpha_{i} \leq 1, \mathrm{i}=1, \ldots, \mathrm{n}$ and $\sum_{i} \alpha_{i}=1$.

The weighted mean of vector $\left\{A_{1}, \ldots, A_{n}\right\}$ is the following $C(\alpha, A)=\left\{\left(x, \mu_{C(\alpha, A)}(x), \eta_{C(\alpha, A)}(x), v_{C(\alpha, A)}(x)\right) \mid x \in X\right\}$ where $\forall x \in X, \quad \mu_{C(\alpha, A)}(x)=\sum_{i=1}^{n} \alpha_{i} \cdot \mu_{A_{i}}(x)$,

$\forall x \in X, \quad \eta_{C(\alpha, A)}(x)=\sum_{i=1}^{n} \alpha_{i} \cdot \eta_{A_{i}}(x)$,

$\forall x \in X, \quad v_{C(\alpha, A)}(x)=\sum_{i=1}^{n} \alpha_{i} \cdot v_{A_{i}}(x)$.

When $\alpha=\alpha_{0}=\{1 / n, \ldots, 1 / n\}$, we obtain the arithmetic mean of vector $\left\{A_{1}, \ldots, A_{n}\right\}$

$C\left(\alpha_{0}, A\right)=\left\{\left(x, \mu_{C\left(\alpha_{0}, A\right)}(x), \eta_{C\left(\alpha_{0}, A\right)}(x), v_{C\left(\alpha_{0}, A\right)}(x)\right) \mid x \in X\right\}$

where $\forall x \in X, \quad \mu_{C\left(\alpha_{0}, A\right)}(x)=\frac{1}{n}\left(\sum_{i=1}^{n} \mu_{A_{i}}(x)\right)$,

$\eta_{C\left(\alpha_{0}, A\right)}(x)=\frac{1}{n}\left(\sum_{i=1}^{n} \eta_{A_{i}}(x)\right), v_{C\left(\alpha_{0}, A\right)}(x)=\frac{1}{n}\left(\sum_{i=1}^{n} v_{A_{i}}(x)\right)$.

Analogously, we can define the weighted mean and the arithmetic mean of vector of IvPFS.

These aggregation operators would be used in the multicriteria decision making problems.

\section{PICTURE FUZZY RELATIONS}

Fuzzy relations are one of most important notions of fuzzy set theory and fuzzy systems theory. The Zadeh' composition rule of inference [6],[11] is a well-known method in approximation theory and inference methods in fuzzy control theory. Intuitionistic fuzzy relations were received many results by researches [4], [5] and [10]. Xu [14] defined some new intuitionistic preference relations, such as the consistent intuitionistic preference relation, imcomplete intuitionistic preference relation and studied their properties. Thus, it is necessary to develop new approaches to issues, such as multi-period investment decision making, medical diagnosis, personnel dynamic examination, and military system efficiency dynamic evaluation. In this section we shall present some preliminary results on picture fuzzy relations.

\section{A. Some definitions for intuitionistic relation}

Let $X, Y$ and $Z$ be ordinary non-empty sets. Definition 13: [4] An intuitionistic fuzzy relation is an intuitionistic fuzzy subset of $X \times Y$, i.e. is an expression given by

$$
R=\left\{\left((x, y), \mu_{R}(x, y), v_{R}(x, y)\right) \mid x \in X, y \in Y\right\},
$$


where $\mu_{R}: X \times Y \rightarrow[0,1] \quad, v_{R}: X \times Y \rightarrow[0,1]$

satisfy the following condition:

$0 \leq \mu_{R}(x, y)+\nu_{R}(x, y) \leq 1 \quad$ for every $\quad(x, y) \in(X \times Y)$.

The most important properties of intuitionistic fuzzy relations were studied in [4],[5].

An extension for picture fuzzy relations is defined as follows:

Definition 14: A picture fuzzy relation $R$ is a picture fuzzy subset of $X \times Y$, i.e. $R$ given by

$$
R=\left\{\left((x, y), \mu_{R}(x, y), \eta_{R}(x, y), v_{R}(x, y)\right) \mid x \in X, y \in Y\right\}
$$

where $\mu_{R}: X \times Y \rightarrow[0,1], \eta_{R}: X \times Y \rightarrow[0,1]$,

$v_{R}: X \times Y \rightarrow[0,1]$ satisfy the condition

$\mu_{R}(x, y)+\eta_{R}(x, y)+v_{R}(x, y) \leq 1$, for every $(x, y) \in(X \times Y)$.

We will represent by $P F R(X \times Y)$ the set of all the picture fuzzy subsets on $X \times Y$.

Defintion 15: Let $R \in P F R(X \times Y)$. We define the inverse relation $R^{-1}$ between $Y$ and $X$ :

$\mu_{R^{-1}}(y, x)=\mu_{R}(x, y), \quad \eta_{R^{-1}}(y, x)=\eta_{R}(x, y)$,

$v_{R^{-1}}(y, x)=v_{R}(x, y), \quad \forall(x, y) \in(X \times Y)$.

Now we will consider simple properties of PFRs.

Definition 16: Let $R$ and $P$ be two picture fuzzy relations between $X$ and $Y$, for every $(x, y) \in X \times Y$ we can define:

- $R \leq P \Leftrightarrow\left(\mu_{R}(x, y) \leq \mu_{P}(x, y)\right) \&$

$\left(\eta_{R}(x, y) \leq \eta_{P}(x, y)\right) \&\left(v_{R}(x, y) \geq v_{P}(x, y)\right)$

$R \vee P=\left\{\left((x, y), \mu_{R}(x, y) \vee \mu_{P}(x, y), \eta_{R}(x, y) \wedge \eta_{P}(x, y)\right.\right.$,

$\left.\left.v_{R}(x, y) \wedge v_{P}(x, y)\right) \mid x \in X, y \in Y\right\}$

$R \wedge P=\left\{\left((x, y), \mu_{R}(x, y) \wedge \mu_{P}(x, y), \eta_{R}(x, y) \wedge \eta_{P}(x, y)\right.\right.$,

- $\left.\left.\quad v_{R}(x, y) \vee v_{P}(x, y)\right) \mid x \in X, y \in Y\right\}$

- $\quad R_{c}=\left\{\left((x, y), v_{R}(x, y), \eta_{R}(x, y), \mu_{R}(x, y)\right) \mid x \in X, y \in Y\right\}$

Proposition 7: Let $R, P, Q \in P F R(X \times Y)$. Then

(a) $\quad\left(R^{-1}\right)^{-1}=R$, (b) $R \leq P \Rightarrow R^{-1} \leq P^{-1}$

(c1) $(R \vee P)^{-1}=R^{-1} \vee P^{-1}$, (c2) $(R \wedge P)^{-1}=R^{-1} \wedge P^{-1}$

(d1) $R \wedge(P \vee Q)=(R \wedge P) \vee(R \wedge Q)$,

(d2) $\quad R \vee(P \wedge Q)=(R \vee P) \wedge(R \vee Q)$

(e) $\quad R \wedge P \leq R, \quad R \wedge P \leq P$ (f 1) If $(R \geq P) \&(R \geq Q)$ then $R \geq P \vee Q$,

(f 2) If $(R \leq P) \&(R \leq Q)$ then $R \leq P \wedge Q$.

Proof: See [8].

\section{B. Composition of Picture Fuzzy Relations}

In this sub-section we shall present some compositons of PFRs. We shall discuss this important problem for approximation reasoning and fuzzy control in the other papers.

The composition of intuitionistic fuzzy relations is given as follows:

Definition 17: [4] Let $\alpha, \beta, \lambda, \rho$ be t-norms or tconorms not necessarily dual two-two, $\quad E \in \operatorname{IFR}(X \times Y)$ and $P \in \operatorname{IFR}(Y \times Z)$. We will call composed relation $P C E \in \operatorname{IFR}(X \times Z) \quad$ to the one defined by $\left.P \mathbb{C} E=\left\{\left((x, z), \mu_{P \mathbb{C} E}(x, y), v_{P \mathbb{C} E}(x, z)\right) \mid x \in X, z \in Z\right)\right\}$, where $\quad \mu_{P \mathbb{}}(x, z)=\underset{y}{\alpha}\left\{\beta\left[\mu_{E}(x, y), \mu_{P}(y, z)\right]\right\} \quad$, $v_{P C E}(x, z)=\lambda_{y}\left\{\rho\left[v_{E}(x, y), v_{P}(y, z)\right]\right\}$

whenever $0 \leq \mu_{P C E}(x, z)+v_{P C E}(x, z) \leq 1 \quad \forall(x, z) \in X \times Z \quad$. In [4] it was proved that take $\alpha=\vee, \beta$ t-norm, $\lambda=\wedge$ , $\rho$ t-conorm, the composition of intuitionistic fuzzy relations satisfies the largest number of properties.

A composition of picture fuzzy relations is defined as follows:

Definition 18: Let $\alpha=\vee, \beta$ t-norm, $\lambda=\wedge, \rho \mathrm{t}-$ conorm be t-norms or t-conorms not necessarily dual twotwo, $E \in P F R(X \times Y)$ and $P \in P F R(Y \times Z)$. We will call composed relation $P \mathbb{C} E \in P F R(X \times Z)$ to the one defined by

$$
\begin{aligned}
& P \mathbb{C} E=\left\{\begin{array}{l}
\left((x, z), \mu_{P C E}(x, y), \eta_{P C E}(x, z),\right. \\
\left.\left.v_{P C E}(x, z)\right) \mid x \in X, z \in Z\right)
\end{array}\right\} \text {, where } \\
& \mu_{P C E}(x, z)=\underset{y}{\vee}\left\{\beta\left[\mu_{E}(x, y), \mu_{P}(y, z)\right]\right\}, \\
& \eta_{P C E}(x, z)=\underset{y}{\vee}\left\{\beta\left[\eta_{E}(x, y), \eta_{P}(y, z)\right]\right\}, \\
& v_{P C E}(x, z)=\underset{y}{\wedge}\left\{\rho\left[v_{E}(x, y), v_{P}(y, z)\right]\right\},
\end{aligned}
$$

whenever

$0 \leq \mu_{P C E}(x, z)+\eta_{P \subset E}(x, z)+v_{P \subset E}(x, z) \leq 1, \forall(x, z) \in X \times Z$.

The first composition of PFRs is the generalized max-min compostion in fuzzy set theory. 
Definition 19: Let $E \in P F R(X \times Y)$ and

$P \in P F R(Y \times Z)$. We will call max-min composed relation $P \mathbb{C E} \in P F R(X \times Z) \quad$ to the one defined by

$\left.P \mathbb{C} E=\left\{\left((x, z), \mu_{P \mathbb{C E}}(x, y), \eta_{P \mathbb{C} E}(x, z), v_{P \mathbb{C E}}(x, z)\right) \mid x \in X, z \in Z\right)\right\}$

where $\mu_{P \mathbb{C E}}(x, z)=\underset{y}{\vee}\left\{\left[\mu_{E}(x, y) \wedge \mu_{P}(y, z)\right]\right\} \quad$,

$\eta_{P \mathbb{C E}}(x, z)=\underset{y}{\vee}\left\{\left[\eta_{E}(x, y) \wedge \eta_{P}(y, z)\right]\right\} \quad$,

$v_{P \mathbb{C E}}(x, z)=\bigwedge_{y}\left\{\left[v_{E}(x, y) \vee v_{P}(y, z)\right]\right\} \quad$.

The second composition of PFRs is the generalized maxprod composition in fuzzy set theory.

Definition 20: Let $E \in P F R(X \times Y)$ and $P \in P F R(Y \times Z)$. We will call max-prod composed relation

$P C E \in P F R(X \times Z) \quad$ to the one defined by

$\left.P \mathbb{C} E=\left\{\left((x, z), \mu_{P \mathbb{C E}}(x, y), \eta_{P \mathbb{C E}}(x, z), v_{P \mathbb{C E}}(x, z)\right) \mid x \in X, z \in Z\right)\right\}$

, where $\forall(x, z) \in X \times Z$

$\mu_{P \mathbb{C E}}(x, z)=\underset{y}{\vee}\left\{\left[\mu_{E}(x, y) \cdot \mu_{P}(y, z)\right]\right\}$,

$\eta_{P \mathbb{C E}}(x, z)=\underset{y}{\vee}\left\{\left[\eta_{E}(x, y) \cdot \eta_{P}(y, z)\right]\right\} \quad$,

$v_{P \mathbb{C E}}(x, z)=\hat{y}_{y}\left\{\left[v_{E}(x, y)+v_{P}(y, z)-v_{E}(x, y) \cdot v_{P}(y, z)\right]\right\} \quad$.

\section{IV.CONCLUSIONS}

In this paper, the new notion of picture fuzzy sets was introduced. Then some operations on PFSs and some properties of these operations were presented. In the parts 2 and 3 more operations including the convex combination were proposed and picture fuzzy relations with their compositions were firstly discussed. In the following paper some classes of aggregation operations with applications in decision making problems and picture fuzzy logic operators should be considered.

\section{ACKNOWLEDGMENT}

This research is funded by Vietnam National Foundation for Science and Technology Development (NAFOSTED) under grant number 102.01-2012.14

\section{REFERENCES}

[1] K. Atanassov, "Intuitionistic fuzzy sets”, Fuzzy Sets and Systems, vol.20,1986, pp. 87-96.

[2] K. Atanassov, "More on intuitionistic fuzzy sets", Fuzzy Sets and Systems, vol.33 ,1989, pp. 37-45.

[3] K. Atanassov and G. Gargov, "Interval valued intuitionistic fuzzy sets”, Fuzzy Sets and Systems, vol.31 ,1989, pp. 343-349.

[4] P. Burilo and H. Bustince, "Intuitionistic fuzzy relations. (Part I)", Mathware and Soft Computing , vol.2, 1995, pp. 5-38.

[5] H. Bustince, "Construction of intuitionistic fuzzy relations with predetermined properties”, Fuzzy Sets and Systems, vol.109, 1996, pp.379-403.

[6] B.C. Cuong and N.D. Phuoc (Ed.), Fuzzy Systems, Neural Networks and Applications, .Science and Technology, Second Ed., 2006 (in Vietnamese).

[7] B.C.Cuong, "Picture fuzzy sets - first results. Part 1", Seminar “ Neuro-Fuzzy Systems with Applications”, Preprint 03/2013, Institute of Mathematics, Hanoi, May 2013.

[8] B.C.Cuong, "Picture fuzzy sets - first results. Part 2", Seminar “ Neuro-Fuzzy Systems with Applications”, Preprint 04/2013, Institute of Mathematics, Hanoi, June 2013.

[9] B.C. Cuong, T.H.Anh and B.D.Hai , "Some operations on type-2 intuitionistic fuzzy sets", J. Computer Science and Cybernetics, vol.28, 2012, pp. 274-283.

[10] G. Deschrijver and E.Kerre , "On the composition of intuitionistic fuzzy relations”, Fuzzy Sets and Systems, vol.136,2003, pp.333-361.

[11] J. Fodor and M.Roubens, Fuzzy preference modeling and multicriteria decision support, Kluwer Academic Pub., London,1994.

[12] T.H.Nguyen and E.Walker, A first course in fuzzy logic, Chapman\& Hall/CRC, Boca Raton, 2000.

[13] E. Szmidt and J. Kacprzyk, "Distances between intuitionistic fuzzy sets”, Fuzzy Sets and Systems. vol.114, 2000, pp.505-518.

[14] Z.S. Xu, "Intuitionistic preference relations and their application in group decision making”, Information Science, vol.177,2007,pp.23632379

[15] L.A.Zadeh, "Fuzzy Sets”, Information and Control , vol.8, 1965, pp. 338-353. 